\title{
典型半干旱区干旱胁迫作用对春小麦蒸散 及其作物系数的影响特征
}

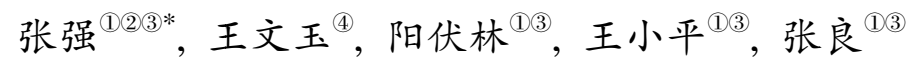

(1) 中国气象局兰州干旱气象研究所, 甘肃省干旱气候变化与减灾重点实验室, 中国气象局干旱气候变化与减灾重点开放实验室, 兰州 730020 ;

(2) 甘肃省气象局, 兰州 730020 ;

(3) 兰州大学大气科学学院, 兰州 730000 ;

(4) 中国气象局气象干部培训学院湖北分院, 武汉 430074

* 联系人, E-mail: zhangqiang@cma.gov.cn, nigzhang@sina.com.cn

2014-11-27 收稿, 2015-02-06 接受, 2015-05-14 网络版发表

国家重点基础研究发展计划(2013CB430206)和国家自然科学基金重点项目(40830957)资助

摘要 蒸散量是地表水分循环的重要分量, 与农业和生态系统的水分需求密切相关, 是水资源 规划和管理必须考虑的重要因素之一。虽然对湿润区作物蒸散计算模型研究已经相对比较成熟, 但由于千旱和半干旱区作物蒸散量受干旱胁迫的显著影响, 以往建立的湿润区作物蒸散模型和 作物系数并不适用对其作物蒸散量的估算, 而目前对这一问题的研究却十分有限. 利用位于黄 土高原典型半千旱区的“定西干旱生态环境综合科学试验站”春小麦农田的蒸渗仪、蒸发皿、超 声涡动仪的观测资料及常规气象观测资料相结合, 分析了该地区实际蒸散量与 FAO 推荐作物系 数估算的蒸散量之间的差异性及其随干旱胁迫度的变化关系. 研究了该地区春小麦参考蒸散量 与蒸发皿蒸发量比值和实际蒸散量与蒸发皿蒸发量比值随干旱胁迫度的变化特征及春小麦作物 系数对干旱胁迫度的响应规律, 并对 FAO 推荐值的作物系数进行了改进. 分析发现, 由于半干 旱区作物蒸散受干旱胁迫影响较大, FAO 推荐作物系数估算的蒸散量与实际蒸散量相差很显著, 实际作物系数对干旱胁迫度的依赖很强, 随干旱胁迫度增加显著减少, 但其敏感性在干旱胁迫 度约达到 0.7 后明显降低. 而且, 改进的作物系数在作物生长初期和发育期远远低于 FAO 的推 荐值和 Kumar 修正值, 改进的作物系数估算的春小麦蒸散量也明显比后两者估算的更接近实际 观测值, 与观测值线性拟合的系数为 0.98 , 决定系数能够达到 0.45 , 标准误差也仅为 $0.85 \mathrm{~mm}$, 对半干旱地区春小麦蒸散量的估算效果比较理想.

\section{关键词}

半于旱区 干旱胁迫度 作物蒸散量 作物系数 模型改进
蒸散是陆面水分循环的重要分量和水分消耗的 主要途径, 直接影响农业和生态系统的需水特征, 是水资源管理和规划必须考虑的重要因素之一 ${ }^{[1]}$. 虽然, 目前有不少研究 ${ }^{[2,3]}$ 用蒸发血观测的蒸发量对 全球陆面蒸散能力的分布特征和和变化趋势做了比 较广泛的分析, 但由于长期以来一直缺乏简便易行 的直接观测陆面实际蒸散的方法, 人们对全球陆
面实际蒸散的认识却十分有限. 所以，近几十年来 发展了不少蒸散量估算模型 ${ }^{[4]}$, 但它们距离真正满 足估算全球不同区域陆面蒸散量的需求仍然有很大 差距, 大多数蒸散模型的估算误差比较大. 因此, 长 期以来国际学术界对作物蒸散模型的改进工作一直 在进行.

理想的作物蒸散模型应该能够充分描述影响蒸 
散过程的各种因素, 但由于对蒸散过程影响机理的 认识所限, 以往的蒸散模型大多只考虑了蒸散过程 的部分因素. 众所周知, 作物蒸散是土壤和植被的液 态水分子从液面逃逸进人大气并被扩散输送的过 程 ${ }^{[5]}$. 具体而言, 就是当土壤和植被表面无规则运动 的液态水分子的动能大于飞出所需克服的液态水分 子间的内聚力或土壤粒子和叶面对水分子的吸附力 时 $^{[6,7]}$, 这些水分子就会脱离液面而飞人大气, 并作 为水汽随大气运动扩散和输送, 从而实现蒸散过程. 这实质上是一个比较复杂的过程 ${ }^{[8]}$, 其影响因素不仅 包括温度、饱和水汽压差、风速与湍流强度等局地气 象条件 ${ }^{[9]}$, 而且还涉及到土壤和植被特征等环境因 素 ${ }^{[10]}$. 但从力学本质上讲, 作物蒸散过程实际上就 是土壤和植被表面液态水分子的约束力与克服约束 力的外强迫力之间的动态平衡过程 ${ }^{[1,12]}$. 因此, 影响 蒸散过程的本质因素主要是水分子的约束力和外强 迫力. 作物蒸散的外强迫力实质上就是大自然对液 态水分子逃逸扩散提供的能量和环境条件, 主要由 表面温度、空气湿度和大气扩散能力等局地气候条件 所决定, 而水分子的约束力是指水分子间吸引力及 土壤粒子对水分子或植物体对水分子的吸附力, 主 要由土壤理化性质、植物生理生态特征及土壤和植被 的干燥程度决定.

实际上, 国际上对作物蒸散模型的发展和完善 经历了一个比较长过程. 最初建立的蒸散估算模型 主要基于影响蒸散过程的温度、风速、饱和水汽压差 等局地气象条件 ${ }^{[13]}$, 基本只考虑了克服水分子约束 力的外强迫力因素, 这实际上只表征了蒸散的气候 强迫力或蒸散能力的大小, 并不能代表真正的蒸散 量. 由此, 在 20 世纪 40 年代Penman等人 ${ }^{[13]}$ 提出了潜 在作物蒸散量的概念 ${ }^{[14]}$, 特指水分供应充足条件下 大范围绿色植被全覆盖表面的蒸散量. 而后, 为了避 免对植被选择的随意性的影响, 20世纪70年代又提出 了专门针对不缺水的高度一致、生长旺盛、完全覆盖 地面的 $12 \mathrm{~cm}$ 高的绿色矮秆作物 (类似于绿草地)的蒸 散量计算模型 ${ }^{[15]}$, 这就是所谓的参考作物蒸散估算 模型, 并陆续发展了一些不同的参考作物蒸散量估 算模型 ${ }^{[16,17]}$, 其中Penman-Monteith参考蒸散估算模 型是目前被最为广泛接受的模型 ${ }^{[18]}$, 它们都在一定 程度上考虑了植被对气候强迫力的作用. 考虑到不 同作物的生理生态差异对作物蒸散过程的液态分子 约束力的影响, 后来的研究又通过对不同作物类型
参考蒸散量的修正来估算作物实际蒸散量 ${ }^{[19]}$, 通常 将这个修正系数称为作物系数 ${ }^{[20]}$, 这以后作物系数 就成了估算作物蒸散量的关键参数. 人们已经认识到, 作物系数主要与作物类型、生长阶段、生长高度和分 布密度等多种因素有关 ${ }^{[21]}$, 并且已经为确定科学合理 的作物系数做出了大量研究 ${ }^{[22]}$. 目前, 国际科学界普 遍认可联合国粮食及农业组织(Food and Agriculture Organization, FAO)给出的作物系数曲线的设计思路, 并且FAO已经在综合国际上主要研究成果基础上专门 推荐了一套针对不同作物的作物系数供全球使用 ${ }^{[23]}$. 最近, 还有一些科学家考虑了地理因素对蒸散的影响, 对FAO推荐的作物系数进行了一定修改 ${ }^{[24,25]}$, 给出了 更加适合复杂地形的作物系数.

然而, 作物系数不只受作物生理生态特征影响, 还会受土壤理化特性和水分特征的作用. 尤其, 土壤 水分含量不仅是蒸散过程必要的物质基础, 而且其 亏缺程度即干旱胁迫程度会显著影响土壤和植被中 液态水分子的内聚力或吸附力即蒸散过程需要克服 的液态水分子约束力大小 ${ }^{[26]}$, 直接关系到作物蒸散 过程对能量和环境条件即气候强迫力的依赖规律 ${ }^{[27]}$, 对蒸散量大小具有十分关键的作用 ${ }^{[28]}$. 在干旱和半 干旱区, 由于土壤和植物大多数时候处于水分亏缺 状态, 干旱胁迫基本上属于气候常态, 干旱胁迫对作 物蒸散的作用非常突出 ${ }^{[29]}$, 在作物系数中应该充分 考虑土壤水分亏缺即干旱胁迫的作用. 春小麦是我 国黄土高原半干旱区主要作物品种, 其生长过程对 土壤水分的依赖性尤其显著, 干旱胁迫作用必然是 影响其作物系数和蒸散过程的重要因素.

但是, 以往确定的作物系数大都基于土壤水分 条件比较充足即不受水分亏缺影响情况下的作物蒸 散过程, 而对干旱胁迫下作物蒸散规律的研究并不 多见，有关干旱胁迫对春小麦等主要作物的作物系 数的影响特征更是缺乏认识, 这严重制约了干旱和 半干旱区农田作物蒸散量的准确估算. 鉴于此, 本文 试图利用位于我国黄土高原典型半干旱区的“定西干 旱生态环境综合科学试验站”在2010 2012年春小麦 农田的综合观测试验资料, 分析观测蒸散、参考蒸散 和蒸发血蒸发量及其比值随干旱胁迫度的变化特征. 并在此基础上, 研究春小麦作物系数对干旱胁迫的 依赖关系, 改进和修正FAO推荐的作物系数, 为半干 旱区春小麦农田蒸散量的准确估算和干旱监测提供 科学依据. 


\section{1 研究资料与计算方法}

\section{1 研究资料}

本文资料来自“定西干旱生态环境综合科学试验 站”的观测试验, 该试验站是“黄土高原陆面过程试 验研究(LOPEX)”的代表性观测站之一 ${ }^{[30]}$, 是我国开 展大型蒸渗仪观测时间最长的观测站之一. 该站地 处甘肃定西市郊区 $\left(35^{\circ} 35^{\prime} \mathrm{N}, 104^{\circ} 37^{\prime} \mathrm{E}\right)$, 属于黄土高 原西南部, 海拔高度 $1896.7 \mathrm{~m}$, 其年平均降水量和年 平均蒸发量分别约为 386 和 $1200 \mathrm{~mm}$, 为典型半干旱 气候区. 该站地貌具有典型的丘陵地貌特征, 观测场 为比较平坦的农田, 主要种植春小麦和马铃薯等耐 旱作物 (图S1). 春小麦播种和收割日期分别在3月中 旬和7月中旬, 全生育期为 $130 \mathrm{~d}$ 左右. 结合小麦的生 育期持续日数和FAO推荐的各阶段的作物系数值, 划分 3 月 1 日 4月 4日为生长初期阶段 (播种-出苗期), 推荐作物系数为 $0.3,4$ 月 5 日 6月 15 日为发育期阶段 (出苗-开花期), 推荐的作物系数从 $0.3 \sim 1.15$ 线性递增, 6月 16 7月 5日为生长中期阶段 (开花-乳熟期), 推荐作 物系数为 $1.15,7$ 月 6 日 7月 31 日为生长末期阶段(乳熟成熟期), 推荐作物系数为从 $1.15 \sim 0.25$ 线性递减.

研究资料主要包括2010 2012年的实际蒸散量资 料、蒸发皿蒸发量资料、近地层水汽通量资料、多层 土壤湿度和温度资料及风、温、湿常规气象资料. 实 际蒸散量用L-G大型称重式蒸渗仪 ${ }^{[31]}$ 观测, 观测精度 为 $0.1 \mathrm{~mm}$, 灵敏度为 $0.01 \mathrm{~mm}$, 经过修正后精度能达 到0.03 mm左右; 蒸发血蒸发量观测根据季节分别采 用小型蒸发血和E601大型蒸发桶观测, 小型蒸发血 口径为 $20 \mathrm{~cm}$, 大型蒸发桶口面积为 $0.3 \mathrm{~m}^{2}$, 观测精 度为 $0.1 \mathrm{~mm}$; 近地面层水汽通量观测采用超声涡动 通量仪, 该仪器为Compbell公司生产的CSAT3型号, 架设在 $2.5 \mathrm{~m}$ 高度; 土壤温度测量采用铂电阻温度计, 分别埋设在地表 $5,10,20,30,50$ 和 $80 \mathrm{~cm}$ 深度; 土壤 湿度观测采用TDR土壤水分仪, 分别埋设在 10,20 , 30,50 和 $80 \mathrm{~cm}$ 深度. 在以往的研究工作中 ${ }^{[32,33]}$ 已对 这些观测仪器布设情况进行了比较详细的说明, 其 技术指标也已有文献 ${ }^{[34,35]}$ 做过介绍.

对超声脉动仪观测的原始资料进行了系统的质 量控制 ${ }^{[36]}$ : (1) 为了消除噪音和奇异值干扰, 当资料 时间序列样本值与相邻样本值的差大于所有相邻样 本值之差的总体标准差的 4 倍时, 该资料样本值作无 效处理. (2) 为了消除降水对感应头的影响, 剔除了
降水时及其前后各 $1 \mathrm{~h}$ 数据. 并利用查表法(LUT)对 资料的极个别缺漏做了插补 ${ }^{[37]}$.

\section{2 计算方法}

一般, 参考蒸散量利用Penman-Monteith公式计 算 ${ }^{[38]}$

$$
E_{\text {ref }}=\frac{0.408 \Delta\left(R_{n}-G\right)+\gamma \frac{900}{T+273} u_{2}\left(e_{\mathrm{s}}-e_{\mathrm{a}}\right)}{\Delta+\gamma\left(1+0.34 u_{2}\right)},
$$

式中, $E_{\mathrm{ref}}$ 是参考蒸散量, 单位为 $\mathrm{mm} ; R_{\mathrm{n}}$ 是表面净辐 射, 单位为 $\mathrm{MJ} \mathrm{m} \mathrm{m}^{-2} \mathrm{~d}^{-1} ; G$ 是土壤热通量, 单位为 $\mathrm{MJ} \mathrm{m}^{-2} \mathrm{~d}^{-1} ; T$ 是 $2 \mathrm{~m}$ 高处气温, 单位为 ${ }^{\circ} \mathrm{C} ; u_{2}$ 是 $2 \mathrm{~m}$ 高 处风速, 单位为 $\mathrm{m} \mathrm{s}^{-1} ; e_{\mathrm{s}}$ 和 $e_{\mathrm{a}}$ 分别是饱和水汽压和实 际水汽压, 单位为 $\mathrm{kPa} ; \Delta$ 表示水汽压/温度斜率, 单位 为 $\mathrm{kPa}{ }^{\circ} \mathrm{C}^{-1} ; \gamma$ 是干湿表常数, 单位也为 $\mathrm{kPa}{ }^{\circ} \mathrm{C}^{-1}$. 这 些变量均可以直接观测或计算. 对于某种作物而言, 其蒸散量可用下式估算 ${ }^{[39,40]}$ :

$$
E_{\mathrm{est}}=k_{c-\mathrm{FAO}} \times E_{\mathrm{ref}},
$$

式中, $E_{\mathrm{est}}$ 是估算的蒸散量, 单位为 $\mathrm{mm} ; k_{c-\mathrm{FAO}}$ 为 $\mathrm{FAO}$ 推荐的作物系数 ${ }^{[23]}$, 因作物种类而异. 具体而言, 其 为随生育阶段变化的系数曲线 ${ }^{[41]}$, 可以表示为

$$
k_{c-\mathrm{FAO}}=\left\{\begin{array}{c}
K_{c-\mathrm{FAO}-\mathrm{ini}}, \quad t_{\mathrm{ini}-s} \leqslant t \leqslant t_{\text {ini- } f} \\
K_{c-\mathrm{FAO}-\mathrm{ini}}+\frac{\left(K_{c-\mathrm{FAO}-\mathrm{mid}}-K_{c-\mathrm{FAO}-\mathrm{ini}}\right)}{\left(t_{\mathrm{dev}-f}-t_{\mathrm{dev}-s}\right)}\left(t-t_{\mathrm{dev}-s}\right), \\
t_{\mathrm{dev}-s} \leqslant t \leqslant t_{\mathrm{dev}-f} \\
K_{c-\mathrm{FAO}-\mathrm{mid}}, \quad t_{\mathrm{mid}-s} \leqslant t \leqslant t_{\mathrm{mid}-f} \\
K_{c-\mathrm{FAO}-\mathrm{end}}-\frac{\left(K_{c-\mathrm{FAO}-\mathrm{mid}}-K_{c-\mathrm{FAO}-\mathrm{end}}\right)}{\left(t_{\mathrm{late}-f}-t_{\mathrm{late}-s}\right)}\left(t-t_{\mathrm{late}-s}\right), \\
t_{\text {late }-s} \leqslant t \leqslant t_{\mathrm{late}-f}
\end{array}\right.
$$

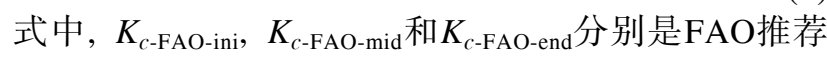
的生长初期、中期和末期的作物系数, 对春小麦而言 它们分为 $0.3,1.15$ 和 $0.25 ; t$ 是年积日, 将全年 365 天排

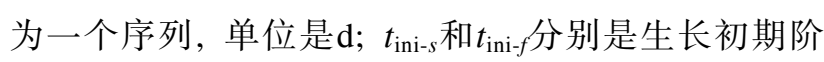
段的起始和终止日期即播种和出苗的时间, 分别为 第60和94天 (3月 1 日和 4 月 4 日); $t_{\mathrm{dev}-s}$ 和 $t_{\mathrm{dev}-f}$ 分别是发 育期阶段的起始和终止日期即出苗和开花的时间,

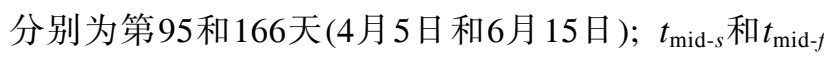
分别是生长中期阶段(旺盛期)的起始和终止日期即 开花和乳熟的时间, 分别为第 167 和 186 天 (6月 16 和 7

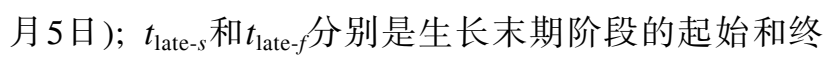


止日期即乳熟和成熟的时间, 分别为第 187 和 212 天 (7月6日和7月31日).

Wright ${ }^{[42]}$ 的研究认为FAO推荐的作物系数在实 际估算蒸散时需要根据当地气候环境特点做进一步 修正. Kumar针对丘陵地形的小气候影响给出了一个 作物系数修正关系 ${ }^{[24]}$, 使作物蒸散量的估算误差缩 小了约 1 倍. 根据该关系, 可将上面的 $K_{c \text {-FAO-mid }}$ 和

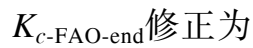

$$
\begin{aligned}
k_{c-\text { Kumar-mid }}= & k_{c-\text { FAO-mid }}+(0.04(u-2) \\
& \left.-0.004\left(R H_{\text {min }}-25\right)\right)\left(\frac{h_{\text {mid }}}{3}\right)^{0.3}, \\
k_{c-\text { Kumar-end }}= & k_{c-\text { FAO-end }}+(0.04(u-2) \\
& \left.-0.004\left(R H_{\text {min }}-25\right)\right)\left(\frac{h_{\text {end }}}{3}\right)^{0.3},
\end{aligned}
$$

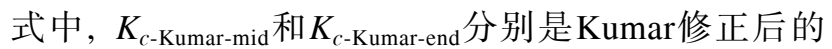
作物生长旺盛期(中期)和末期的作物系数, 其他时段 作物系数可以由此推算 ${ }^{[24]}$; $u$ 是平均风速, 单位为 $\mathrm{m} \mathrm{s}^{-1} ; R H_{\mathrm{min}}$ 是每日最小相对湿度, 单位为 $\% ; h_{\mathrm{mid}}$ 和 $h_{\mathrm{end}}$ 分别是作物生长旺盛期和末期的平均高度, 单位 为 $\mathrm{m}$. 该修正关系应该比较适合黄土高原丘陵地貌.

不过, 实际的作物系数需要通过观测试验来确 定，可以用下式来表示

$$
k_{c-\mathrm{obs}}=\frac{E_{\mathrm{obs}}}{E_{\text {ref }}},
$$

式中, $k_{c-\mathrm{obs}}$ 是实际观测的作物系数; $E_{\mathrm{obs}}$ 是实际观测的 蒸散量, 单位为 $\mathrm{mm}$, 这里用 $\mathrm{L}-\mathrm{G}$ 大型称重式蒸渗仪 的观测值, 这是目前最有效的蒸散量观测方法 ${ }^{[42,43]}$

$$
E_{\mathrm{obs}}=1000 \times \sum_{i=0}^{i=T} \Delta f_{i} /\left(\rho_{\mathrm{w}} \times S_{\mathrm{obs}}\right) \text {, 当 } \Delta f_{i} \leqslant 0,
$$

式中, $\rho_{\mathrm{w}}$ 是水的比重, 单位为 $\mathrm{kg} \mathrm{m}^{-3} ; f_{i}$ 是蒸渗仪的蒸 散盘内水分的瞬时重量值, $\Delta f_{i}$ 为其瞬时变量值, 单位 均为 $\mathrm{kg}$; $i$ 是瞬时值的序列号, 每隔 $5 \mathrm{~min}$ 一个值; $S_{\mathrm{obs}}$ 是观测盘的面积, 单位为 $\mathrm{m}^{2} ; T$ 是每个资料样本采样 时间长度, 单位为 $\mathrm{s}$, 这里取 $0.5 \mathrm{~h}$.

另外, 如果假定近地面层为水汽常通量层, 还可 以用浴动相关法间接观测地表蒸散量 ${ }^{[44]}$ :

$$
E_{\text {ed }}=1000 \times\left(\rho \overline{w^{\prime} q^{\prime}} / \rho_{w}\right) \times \Delta t,
$$

式中, $E_{\mathrm{ed}}$ 是涡动相关法观测的蒸散量, 单位为 $\mathrm{mm} ; \rho$ 为空气密度, 单位为 $\mathrm{kg} \mathrm{m}^{-3} ; q^{\prime}$ 和 $w^{\prime}$ 分别是近地层比 湿和垂直速度脉动, 单位分别为 $\mathrm{g} \mathrm{kg}^{-1}$ 和 $\mathrm{m} \mathrm{s}^{-1}$. 它们 均可由超声脉动仪器直接观测得到.
为了验证对作物蒸散量观测的可靠性，对蒸渗 仪直接观测的蒸散量与浴动相关法间接观测的蒸散 量进行了比较(图S2). 对比结果表明, 蒸渗仪观测的 蒸散量与浴动相关法观测的蒸散量相当一致, 拟合 系数为 0.84 , 二者的决定系数也较高, 达到了 0.72 ; 标准差较小, 仅为 $0.35 \mathrm{~mm}$. 这表明蒸渗仪和涡动相 关法观测的蒸散量都能够比较好地反映作物的实际 蒸散量.

考虑到作物蒸散受干旱胁迫的突出作用, 特定 义了一个能够较好表征干旱胁迫的参数

$$
I_{\text {arid }}=\frac{\left(s_{\mathrm{sa}}-S_{\mathrm{w}}\right)-\left(s_{\mathrm{m}}-S_{\mathrm{w}}\right)}{s_{\mathrm{sa}}-s_{\mathrm{w}}}=1-\frac{s_{\mathrm{m}}-S_{\mathrm{w}}}{S_{\mathrm{sa}}-S_{\mathrm{w}}},
$$

式中, $I_{\mathrm{arid}}$ 是干旱胁迫度; $s_{\mathrm{m}}, s_{\mathrm{w}}$ 和 $s_{\mathrm{sa}}$ 分别是土壤湿度、 调萎系数和田间持水量, 单位均是\%(体积百分数), 在这里田间持水量和调萎系数分别为 $24.39 \%$ 和 $7.47 \%$. 从理论上讲, $I_{\text {arid }}$ 应该在 $0 ~ 1$, 当土壤湿度达到 田间持水量时, 干旱胁迫度为 0 , 表示不受干旱胁迫 影响, 气候蒸散力可以得到完全发挥; 当土壤湿度小 于田间持水量时，气候蒸散力会受到干旱胁迫的约 束; 土壤湿度越小, 干旱胁迫就越强, 对蒸散的约束 就越突出; 当土壤湿度等于调萎系数时, 干旱胁迫达 到最强，蒸散基本被彻底抑制.

\section{2 干旱胁追对作物蒸散的影响}

一般，都用式(2)的作物蒸散模型来估算作物蒸 散量, 但由于其用到的作物系数大多是在比较理想 的环境和水分供应充足的条件下得到，这种方法估 算的作物蒸散量在很多情况并不可靠，尤其在受干 旱胁迫较严重的干旱和半干旱地区其可靠性更是值 得怀疑.

为了了解这一问题, 在图1中对FAO推荐作物系 数估算的蒸散量与黄土高原半干旱区定西实际观测 的蒸散量进行了对比. 该图很清楚地表明, 用FAO作 物系数估算的作物蒸散量与实际观测的蒸散量相差 十分显著, 拟合系数为 1.55 , 标准差为 $2.54 \mathrm{~mm}$, 相 对误差高达 $120 \%$ 左右，观测的蒸散量平均不到模型 估算的一半; 两者的相关性也较低, 决定系数仅为 0.12. 该蒸散模型明显高估了作物蒸散量, 而且实际 蒸散量越小高估程度越明显, 最大时能高估 $5.4 \mathrm{~mm}$. 这表明, 在半干旱地区, 直接用FAO推荐的作物系数 构建的作物模型来估算作物蒸散量并不合适. 


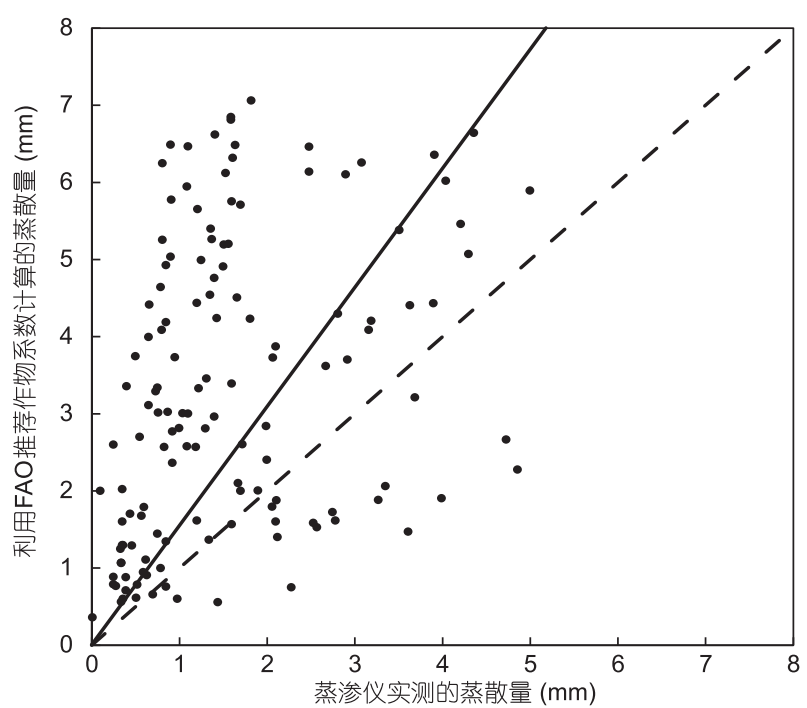

图 1 黄土高原半干旱地区 FAO 推荐作物系数估算的春小麦蒸散量 与观测的蒸散量的对比

Figure 1 The comparison of estimated evapotranspiration using the FAO recommended crop coefficient and the actual evapotranspiration in semiarid region

那么, 造成半干旱区模型估算的蒸散量与观测 的实际蒸散量之间不一致的主要的原因是什么呢? 由图2给出的黄土高原半干旱区春小麦实际蒸散量随 干旱胁迫度的变化关系可以看出, 虽然由于受局地 气象条件变化影响, 观测的作物蒸散量与干旱胁迫 度的关系比较离散一些, 但其被干旱胁迫度主导的 趋势却十分突出, 蒸散量明显随干旱胁迫度的增强 而减小. 在干旱胁迫度为 0 即基本没有干旱胁迫时, 日作物蒸散量接近于 $4 \mathrm{~mm}$, 但在干旱胁迫最强时日 作物蒸散量几乎减少到 $0.5 \mathrm{~mm}$. 这种现象是比较容 易理解的: 在干旱胁迫下, 不仅作物覆盖率较低, 蒸 发在蒸散过程的作用更突出, 而且作物叶面和土壤

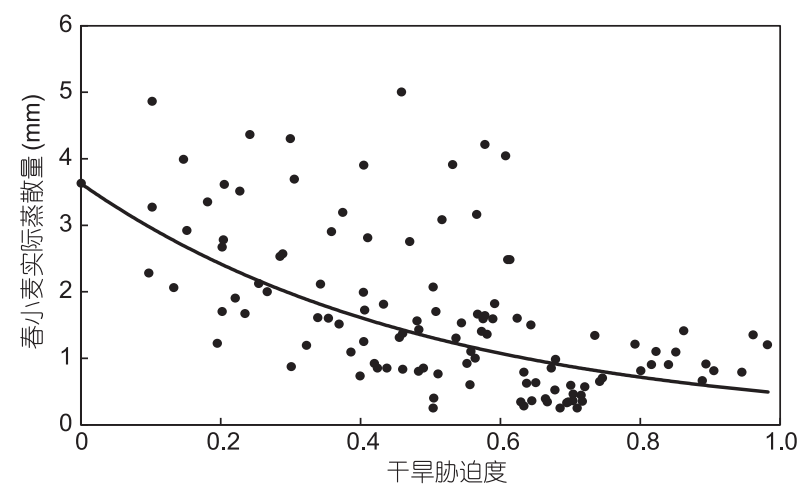

图 2 半干旱区春小麦实际蒸散量随干旱胁迫度的变化关系

Figure 2 The changes of actual evapotranspiration with the degree of drought stress in semiarid region
粒子对水分子的吸附力更强，会大大制约了水分的 蒸散过程. 可见, 在半干旱区, 干旱胁迫对蒸散的控 制作用很强，而以往的经典蒸散估算模型基本没有 考虑这种作用，所以必然会出现模型估算量与实际 观测值的显著差异.

不过，作物蒸散量在干旱胁迫度较低时对干旱 胁迫作用的响应更敏感, 而在干旱胁迫度达到 0.7 以 后, 作物蒸散量对干旱胁迫度的敏感性有所降低, 这 说明在干旱胁迫度达到临近值后干旱胁迫作用会表 现出一定的收玫性.

为了能够更好地突出干旱胁迫和地表因素对蒸 散的作用, 分别用参考蒸散与蒸发血蒸发的比值和 实际蒸散与蒸发血蒸发的比值作为作物蒸散参数, 它们能够在一定程度上消除局地气象条件变化对作 物蒸散的影响. 其中, 参考蒸散与蒸发血蒸发的比值 能够表征植被和土壤本身对蒸散的影响, 而实际蒸 散与蒸发血蒸发的比值可以进一步表征干旱胁迫对 蒸散过程的影响. 图3(a)中给出了该地区参考蒸散/ 蒸发血蒸发随干旱胁迫度的变化趋势可以看出, 参
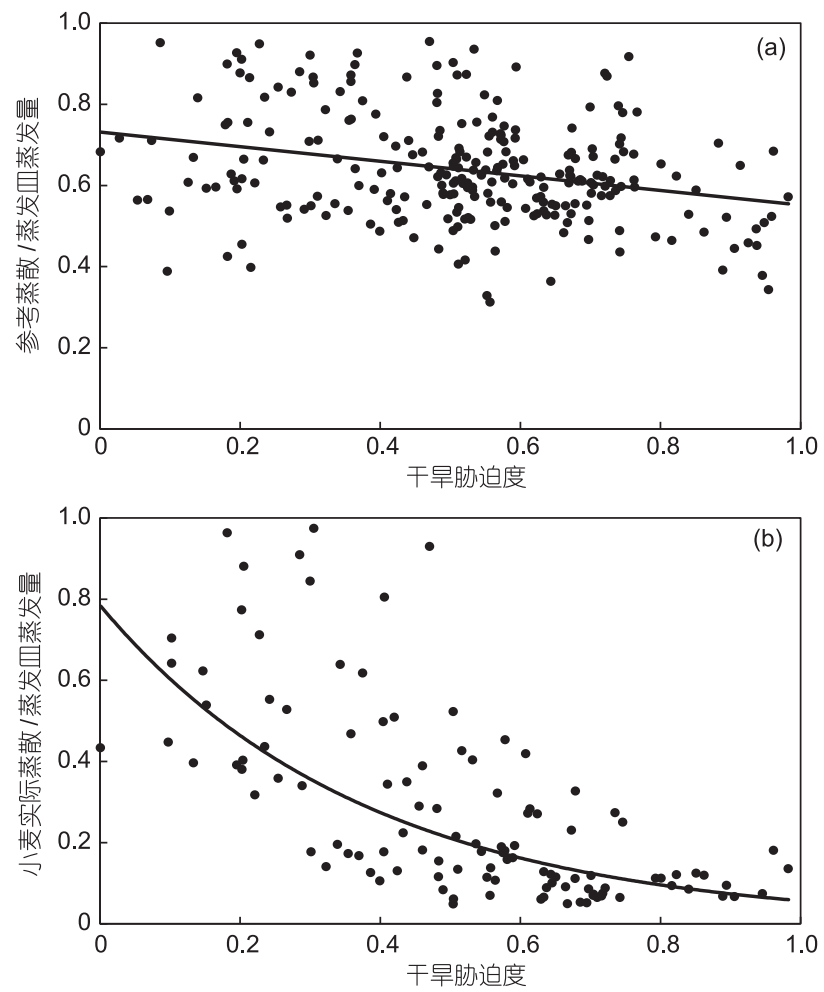

图 3 半干旱区春小麦参考蒸散/蒸发皿蒸发(a)和实际蒸散/蒸发皿蒸 发(b)随干旱胁迫度的变化关系

Figure 3 The changes of reference evapotranspiration/pan evaporation (a) and actual evapotranspiration/pan evaporation (b) with the degree of drought stress in semiarid region 
考蒸散/蒸发血蒸发的比值在没有干旱胁迫时(干旱 胁迫度在 0 附近)大约在 0.73 , 这说明仅仅植被和土壤 本身就可以使蒸散量限制在蒸发力的 $73 \%$ 左右. 而 且, 随着干旱胁迫度的增强, 参考蒸散/蒸发血蒸发 还会缓慢减少, 在干旱胁迫最强时减小到了 0.59 左 右. 这是由于蒸发血的蒸发水面较小, 蒸发的局地气 象条件受周围环境的显著影响, 干旱胁迫度的加强 会导致局地气象条件向有利于蒸发的趋势发展, 从 而使蒸发皿蒸发量有所增大.

从图3(b)与(a)对比表明, 虽然在没有干旱胁迫时 即干旱胁迫度在 0 附近时, 实际蒸散/蒸发血蒸发的比 值也接近 0.73 . 但当干旱胁迫度增加时, 实际蒸散/ 蒸发血蒸发的比值要远比参考蒸散/蒸发血蒸发的比 值减少的迅速, 在干旱胁迫度达到 0.7 时就已减小到 了 0.1 左右, 在干旱胁迫度最大时几乎接近于 0 . 这说 明干旱胁迫能够显著增强植被和土壤对水分子的约 束力, 从而有效抑制蒸发力的发挥.
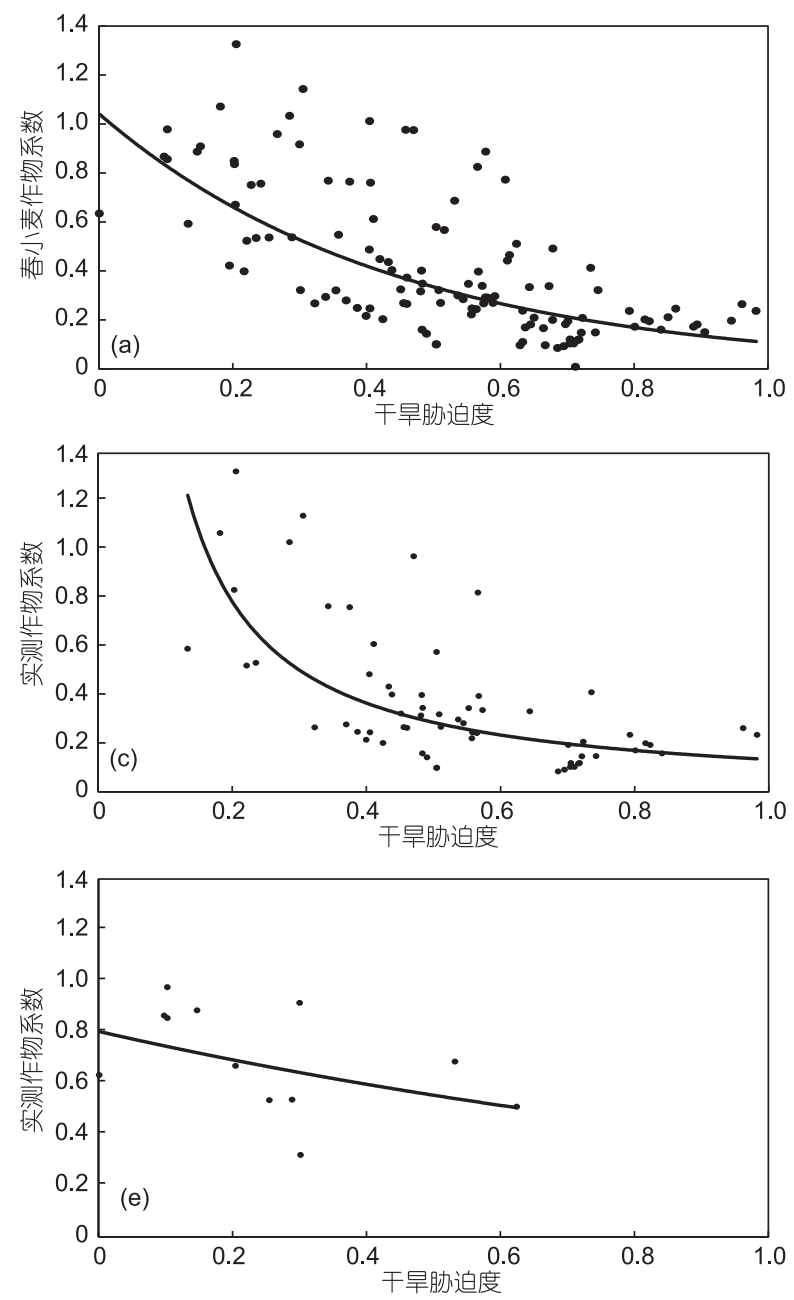

\section{3 作物系数与干旱胁追的关系}

根据作物蒸散模型的原理，干旱胁迫对半干旱 区春小麦蒸散过程的显著作用可以通过作物系数有 效体现出来. 根据式(6), 春小麦实际作物系数可由 实际蒸散/参考作物蒸散得到，结合图2春小麦实际蒸 散量随干旱胁迫度的变化, 得到并给出黄土高原半 干旱区春小麦实际作物系数随干旱胁迫度的变化(图 4(a)). 从图4(a)中很容易看出，该地区春小麦作物系 数随干旱胁迫度变化十分明显, 随干旱胁迫度增强 而显著减小. 在没有干旱胁迫时作物系数大约在 1.0 , 在干旱胁迫度达到 0.7 时就减少到了 0.23 左右，随后 减小趋势变得相对缓慢; 在干旱胁迫度最大时, 作物 系数只有 0.1 左右. 而且, 还可以给出作物系数与干 旱胁迫度之间的拟合关系，

$$
k_{c-\text { obs }}=1.0423 \times \mathrm{e}^{-2.2710 \times I_{\text {arid }} \text {, }}
$$

该拟合关系式的决定系数为 0.41 , 均方差仅 0.049 左
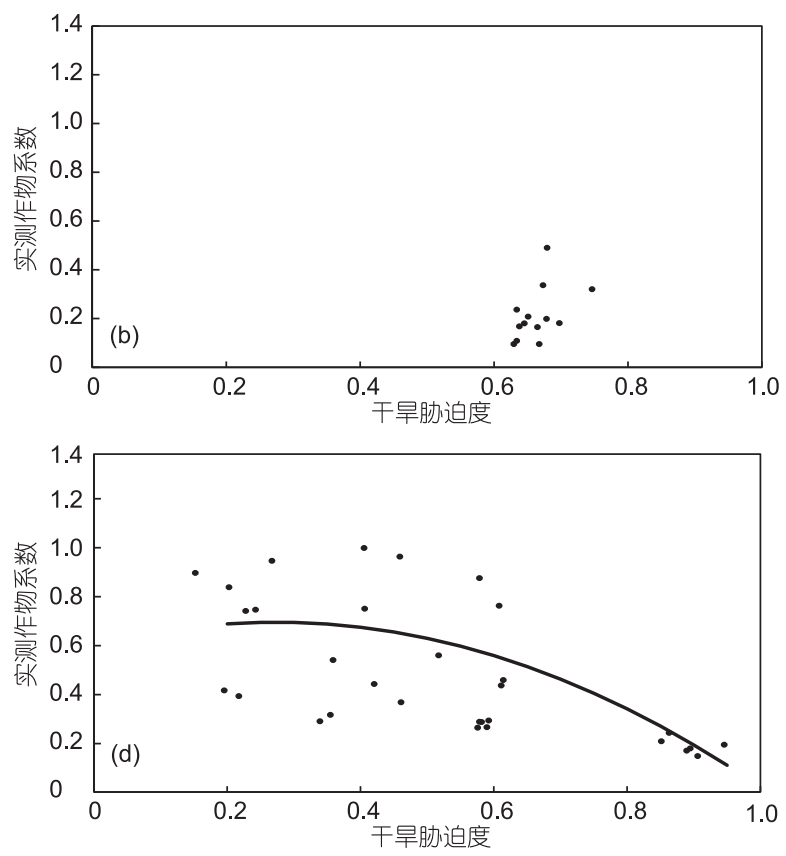

图 4 半干旱区春小麦实际作物系数(a)与干旱胁迫度的关系曲 线及生长初期 $(b) 、$ 发育期 (c)、旺盛期 $(\mathrm{d})$ 和末期 $(\mathrm{e})$ 的实际作物 系数随干旱胁迫度的分布特征

Figure 4 The relationship between measured crop coefficients of spring wheat and the degree of drought stress in semiarid region (a), and the changes of measured crop coefficients in initial stage (b), development period (c), vigorous stage (d) and the growth telophase (e) with the degree of drought stress in semiarid regions 
右. 这说明在半干旱区作物系数对干旱胁迫度具有 很强的依赖性. 然而, 这一问题在以往的作物系数中 大多数时候是被忽视的.

由于作物在生长过程中生理生态特征变化较大, 作物蒸散对干旱胁迫的依赖程度也会有所不同 ${ }^{[45]}$. 图4表明, 在作物生长的4个典型阶段即生长初期、发 育期、旺盛期和末期, 除了在生长初期, 由于干旱胁 迫度变化范围太小看不出其与作物系数的关系而外, 其他 3 个阶段表现出了一些突出的区别, 发育期、旺 盛期和末期拟合关系的决定系数分别为 0.4421 , $0.2537,0.1746, P$ 值均小于 0.05 . 这 3 个阶段相比较而 言, 生长末期作物系数随干旱胁迫变化的影响相对 较小, 发育期随干旱胁迫度变化最显著. 通过拟合曲 线的斜率可以看出, 作物系数在发育期对弱干旱胁 迫更敏感, 而在旺盛期对强干旱胁迫更敏感. 这种表 现特征与作物各个生长阶段的生理生态特征对水分 的依赖程度是基本一致的. 春小麦生长初期、发育 期、旺盛期、末期的日平均需水量分别为 $0.70,2.99$, 4.98 和 $2.93 \mathrm{~mm}$. 生长发育期春小麦完成拔节、孕穗、 抽穗至开花, 是作物生长的关键时期和作物需水关键 期, 所以较弱程度的干旱就很有可能对春小麦的生长 发育产生影响. 至生长旺盛期, 春小麦完成开花-乳 熟, 所需降水量最大, 由于春小麦已经完成初期的生 长发育, 此阶段主要进行灌浆乳熟, 较弱的干旱胁迫 只会对春小麦的产量有较大影响, 而干旱胁迫较强 时则有可能会影响春小麦的生长生存, 因此在旺盛 时期作物系数对强干旱胁迫更加敏感. 春小麦在末 期已近成熟, 需水量相对较小, 作物系数与干旱胁迫 的关系也较发育期和旺盛期不那么明显. 而生长初 期、发育期、旺盛期、末期的日平均蒸散量(耗水量) 分别为 $0.80,1.29,1.80$ 和 $2.05 \mathrm{~mm}$. 末期的蒸散量(耗 水量)较大, 可能是由于该时期为 7 月中下旬, 雨水增 多、温度较高造成的.

上面的式(10)虽然给出了半干旱区春小麦实际 作物系数与干旱胁迫度的依赖关系, 但为了能够与 $\mathrm{FAO}$ 作物系数曲线设计思路相一致, 可以在目前 FAO推荐的春小麦作物系数基础上针对干旱胁迫作 用给出了一个作物系数的修正关系

$$
k_{c-\bmod i f}=k_{c-\mathrm{FAO}} \times f\left(I_{\text {arid }}\right),
$$

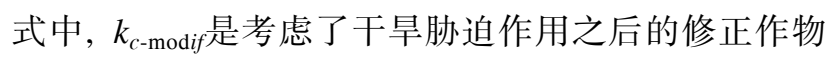
系数, $f\left(I_{\text {arid }}\right)$ 是 $\mathrm{FAO}$ 推荐作物系数的干旱胁迫度修正 函数. 为了突出干旱胁迫度修正函数的变化特征, 可
以将式(11)转化为

$$
f\left(I_{\text {arid }}\right)=k_{c-\text { modif }} / k_{c-\mathrm{FAO}} .
$$

如果将式(10)中实际观测的作物系数作为 $k_{c \text {-modif }}$ 的理想值, 除以各自所处生长阶段期间的FAO推荐 作物系数后进行拟合, 就可以给出如图 5(a)所示的作 物系数的干旱胁迫修正函数随干旱胁迫度的变化曲 线. 由图5(a) 可见, 干旱胁迫修正函数与干旱胁迫度 的相关性较好, 决定系数能够达到 0.38 , 均方差仅为 0.78 , 其指数拟合关系为

$$
f\left(I_{\text {arid }}\right)=\frac{k_{c-\text { mod } i f}}{k_{c-\mathrm{FAO}}}=1.351 \mathrm{e}^{-2.076 \times I_{\text {arid }}} .
$$

该拟合关系表明，干旱胁迫修正函数在没有干 旱胁迫时大约为 1.351 , 与 $\mathrm{FAO}$ 推荐值比较接近, 然
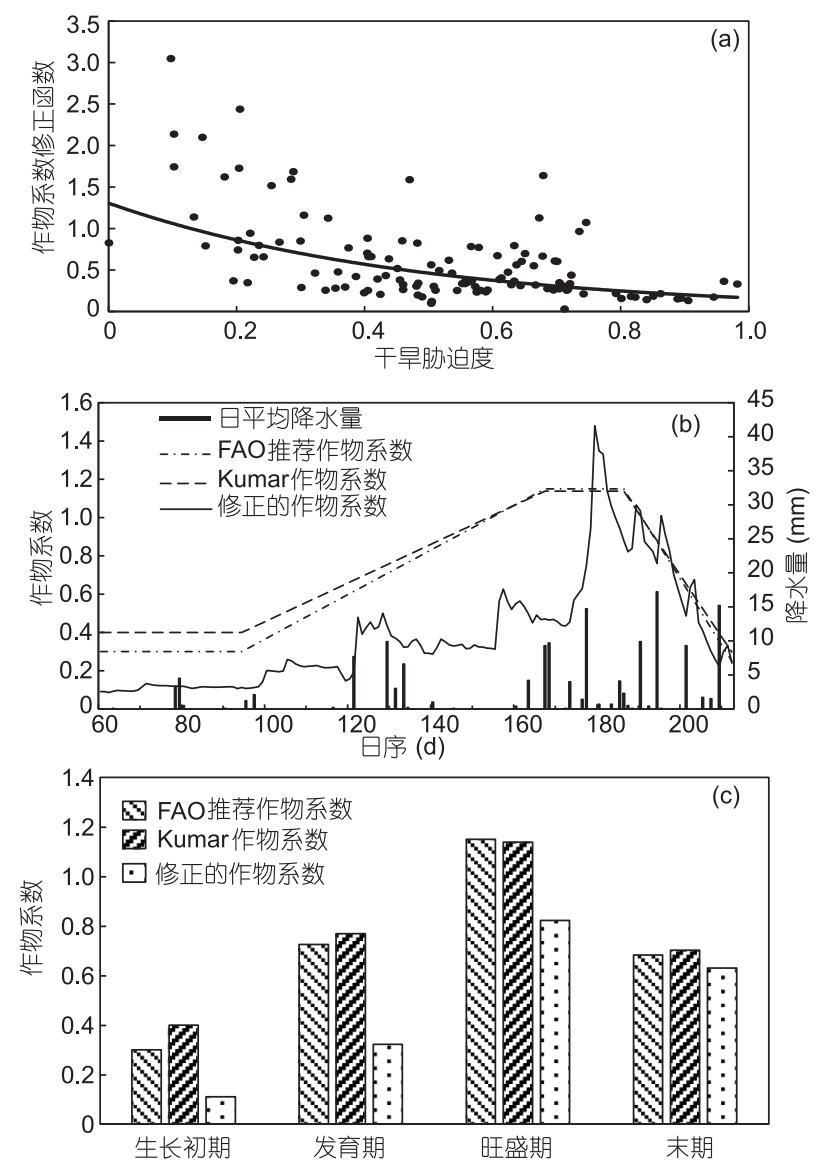

图 5 半干旱区作物系数的干旱胁迫修正函数随干旱胁迫度的变化 关系(a)及不同生育期本文改进的春小麦作物系数与 Kumar 修正作物 系数、FAO 推荐作物系数的变化曲线(b)和平均值(c)对比

Figure 5 The changes of correction function of crop coefficients with the degree of drought stress in semiarid regions (a), and the curves (b) and averages (c) of revised crop coefficients, Kumar crop coefficients and FAO recommended crop coefficients in different growth stages of spring wheat crop 
后逐渐减小, 在干旱胁迫最大时, 几乎减小到 0.16 左 右, 可见其修正幅度是很大的. 不过, 在干旱胁迫小 于 0.7 时修正函数变化比较敏感, 干旱胁迫过大则变 化不太敏感. 对(13)式稍作变化就可以得到半干旱区 春小麦作物系数的修正关系

$$
k_{c-\bmod i f}=k_{c-\mathrm{FAO}} \times 1.351 \mathrm{e}^{-2.076 \times I_{\text {arid }}} .
$$

上式对作物系数的修正, 充分考虑了干旱胁迫 对作物系数的影响机制, 能够更好反映作物系数的 变化特征. 在图 5(b)和(c)中, 对本文改进的春小麦作 物系数与 FAO推荐作物系数和Kumar修正作物系数 在整个生长季的变化曲线(图5(b))及其不同生长期的 平均值(图5(c))对比表明, 本文改进的作物系数虽然 在末期与 FAO推荐的作物系数和Kumar修正的作物 系数比较接近, 但在作物生长初期、发育期和旺盛期 FAO推荐的作物系数和 Kumar修正的作物系数均明 显偏高, 尤其在生长初期和发育期偏高更为显著. 而 且, 在作物发育期, 本文修正的作物系数并没有如 FAO推荐的作物系数和Kumar修正的作物系数一样 立即表现出明显的增加, 而是呈现大致随降水过程 的波动性增加. 生长过程与降水过程同时控制着生 长期作物系数的变化趋势. 可见, 在半干旱区, 干旱 胁迫对作物系数的修正作用是很明显的, 而丘陵地 形对作物系数的修正作用倒并不明显.

为了检验本文改进的作物系数对黄土高原半干 旱区作物蒸散量的估算效果, 利用定西试验站2004 年6月 2005年5月春小麦生长季期间的蒸渗仪、土壤 湿度等数据对改进的作物系数进行检验. 图6分别对 本文改进作物系数、Kumar修正作物系数和FAO推荐 作物系数估算的蒸散量与实测蒸散量进行了相关性 比较. 该图清楚表明, 本文改进的作物系数估算的蒸 散量与观测值比较一致, 它们之间的线性系数达到 了0.98左右, 而Kumar作物系数和FAO作物系数估算 的蒸散量的线性系数分别为 1.81 和 1.77 左右, 相差十 分明显; 它们之间的决定系数也达到了 0.45 , 分别比 Kumar作物系数和FAO作物系数估算的蒸散量高了 0.18 和 0.13 ; 标准误差也仅为 0.85 , 分别比Kumar作 物系数和FAO作物系数估算的蒸散量降低了 1.14 和 $1.10 \mathrm{~mm}$. 与Kumar作物系数和FAO作物系数相比, 本文改进的作物系数估算蒸散量的相对误差分别由 $153 \%$ 和 $134 \%$ 降低到了 $13 \%$ 左右, 明显提高了对作物 蒸散量的估算精度. 当然, 改进后的作物系数估算的 蒸散量仍然要比观测值稍偏低一些, 这说明除了干
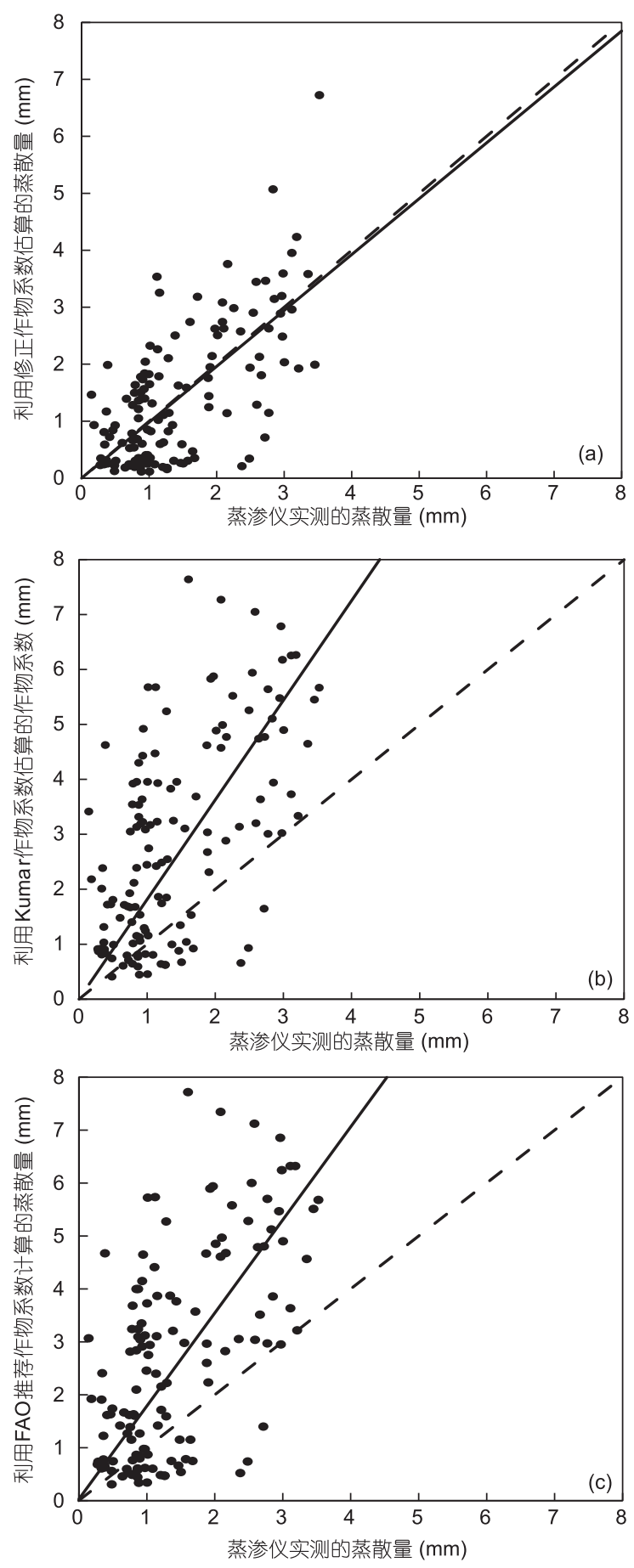

图 6 修正作物系数(a)、Kumar 作物系数(b)和 FAO 推荐作物系数(c) 估算的蒸散量与实测的蒸散量的相关性比较

Figure 6 The comparison of correlation between the estimated evapotranspiration using the revised crop coefficient (a), Kumar crop coefficient (b) and FAO recommended crop coefficient (c) and the measured evapotranspiration

旱胁迫而外，目前还没有考虑到的其他因素也会对 作物系数有一定影响. 


\section{4 结论与讨论}

在黄土高原典型半干旱区, 由于干旱少雨, 土壤 含水量长期处于较低状态, 导致作物覆盖率较低和 作物需水严重亏缺, 干旱胁迫作用十分突出, 由此造 成以往经典的作物蒸散模型估算的作物蒸散量与实 际观测的蒸散量相差比较显著, 线性拟合系数为 1.55 , 决定系数仅为 0.12 , 标准差高达 $2.54 \mathrm{~mm}$, 明显 不适宜直接用来估算该地区作物蒸散量.

在半干旱地区, 春小麦蒸散量随干旱胁迫度变 化十分明显, 大致能从没有干旱胁迫时的 $4 \mathrm{~mm}$ 减少 到干旱胁迫最大时的 $0.5 \mathrm{~mm}$, 作物蒸散量变化明显 受干旱胁迫度控制. 而且, 观测的实际作物系数与干 旱胁迫度的相关性也非常好, 具有较好的指数拟合 关系, 决定系数高达 0.41 , 均方差仅为 $0.049 \mathrm{~mm}$. 作 物系数对干旱胁迫度的敏感性大约在干旱胁迫度小 于 0.7 时比较高, 之后敏感性会明显降低, 表现出一 定的收玫性.

可以在FAO推荐的作物系数的基础上针对干旱 胁迫作用对作物系数进行比较合理的改进, 改进的 作物系数对干旱胁迫度有较好的依赖关系, 决定系 数大约为 0.45 , 均方差仅为 $0.85 \mathrm{~mm}$. 用改进的作物 系数估算的作物蒸散量与实际观测作物蒸散量的标
准差均远比Kumar作物系数和FAO作物系数的小, 标 准误差分别比Kumar作物系数和 $\mathrm{FAO}$ 作物系数估算 的蒸散量降低了 1.14 和 $1.10 \mathrm{~mm}$, 相对误差由大约 153\%降低到了 13\% , 这意味著, 在半干旱区, 干旱胁 迫对作物系数的修正作用是很明显的, 而丘陵地形 对作物系数的修正作用倒并不明显, 本文改进的作 物系数比较适合用来估算作物蒸散量.

本文改进的春小麦作物系数与Kumar修正作物 系数和FAO推荐作物系数明显不同, 除生长末期外 其他 3 个生长阶段的作物系数都要明显低于Kumar修 正作物系数和FAO推荐作物系数. 尤其在作物发育 期, 本文改进的作物系数呈现出随降水过程的波动 性增加, 作物系数变化趋势明显由生长过程和降水 过程同时控制着.

本文虽然针对半干旱区干旱胁迫作用的特征, 对春小麦作物系数受干旱胁迫的影响规律进行了深 人分析, 但由于作物系数受影响的因素比较复杂, 只 考虑干旱胁迫度的作物系数修正关系并不一定能够 完全刻画半干旱区作物系数的影响机制, 这可能是 造成改进的作物系数估算的蒸散量与实际观测值仍 有一定偏差的原因, 这需要在今后考虑建立多影响 因子作物系数修正关系来进一步提高作物蒸散量估 算的准确性.

\section{参考文献}

1 Groisman P Y, Knight R W, Karl T R, et al. Contemporary changes of the hydrological cycle over the contiguous United States: Trends derived from in situ observations. J Hydrometeorol, 2004, 5: 64-85

2 Michael L R, Graham D F. The cause of decreased pan evaporation over the past 50 year. Science, 2002, 298: 1410-1411

3 Fu G B, Charles S P, Yu J J. A critical overview of pan evaporation trends over the last 50 years. Clim Change, 2009, 97: 193-214

4 Schlosser C A, Gao X. Assessing evapotranspiration estimates from the second Global Soil Wetness Project (GSWP-2) simulations. J Hydrometeorol, 2010, 11: 880-897

5 Brutsaert W. Evaporation into the Atmosphere. Dordrecht: D. Reidel Publishing Company, 1982. 299

6 Moore W J. Physical Chemistry. Landon: Longman, 1972

7 Hillel D. Environmental Soil Physics. Manhattan: Academic Press, 1998

8 Monteith J L, Unsworth M H. Principles of Environmental Physics. 2nd ed. London: Edward Arnold, 1990

9 Gong L B, Xu C Y, Chen D L, et al. Sensitivity of the Penman-Monteith reference evapotranspiration to key climatic variables in the Changjiang basin. J Hydrol, 2006, 329: 620-629

10 Andre J C. HAPEX-MOBILMY. Bull Amer Meteor Soc, 1998, 68: 138-144

11 Heitman J L, Horton R, Sauer T J, et al. Sensible heat observations reveal soil-water evaporation dynamics. J Hydrometeorol, 2008, 9: 165-171

12 Trenberth K E, Fasullo J T, Kiehl J. Earth's global energy budget. Bull Amer Meteorol Soc, 2009, 90: 311-323

13 Penman H L. Natural evaporation from open water, bare soil and grass. Proc R Soc London, 1948, A193: 120-146

14 Hargreaves G H, Samani Z A. Estimating of potential evapotranspiration. J Irrig Drain, 1982, 108: 223-230

15 Fan J, Shao M A, Wang Q J. Comparison of many equations for calculating reference evapotranspiration in the Loess Plateau of China (in Chinese). Trans Chin Soc Agric Eng, 2008, 24: 98-102 [樊军, 邵明安, 王全九. 黄土区参考作物蒸散量多种计算方法的比较. 农业工 程学报, 2008, 24: 98-102] 
16 Pereira A R, Pruitt W O. Adaptation of the Thornthwaite scheme for estimating daily reference evapotranspiration. Agric Water Manage, 2004, 66: 251-257

17 Gavilan P, Lorite I J, Tornero S, et al. Regional calibration of Hargreaves equation for estimating reference ET in a semiarid environment. Agric Water Manage, 2006, 81: 257-281

18 Pereira A R, Green S, Nova N A. Penman-Monteith reference evapotranspiration adapted to estimate irrigated tree transpiration. Agric Water Manage, 2006, 83: 153-161

19 Rosenberg N J, Blad B L, Verma S B. Evaporation and evapotranspiration. In: Microclimate-The Biological Environment. New York: Wiley-Interscience, 1983. 209-287

20 Doorenbos J, Pruitt W O. Guidelines for Prediction Crop Water Requirements. Rome: Food and Agriculture Organization of the United Nations, 1977. 1-179

21 Brutsaert W, Stricker H. Advection-aridity approach to estimate actual regional evapotranspiration. Water Resour Res, 1979, 15: 443-450

22 Gonzalez-Dugo M P, Neale C M U, Mateos L, et al. A comparison of operational remote sensing-based models for estimating crop evapotranspiration. Agric For Meteorol, 2009, 149: 1843-1853

23 Allen R G, Pereira L S, Raes D, et al. Crop Evapotranspiration-Guidelines for Computing Crop Water Requirements. Rome: United Nations Food and Agriculture Organization, 1998

24 Rohitashw K, Vijay S, Mahesh K. Development of crop coefficients for precise estimation of evapotranspiration for mustard in Mid Hill Zone-India. Universal J Environ Res Tech, 2011, 4: 531-538

25 Yang G, Duan A W, Sun J S. Crop coefficient and water-use efficiency of winter wheat/spring maize strip intercropping. Field Crops Res, 2009, 111: 65-73

26 Penman H L. Natural evaporation from open water, bare soil and grass. Proc R Soc A, 1948, 193: 120-146

27 Arora V. Modeling vegetation as a dynamic component in soil-vegetation-atmosphere transfer schemes and hydrological models. Rev Geophys, 2002, 40: 1006-1011

28 Gavilán P, Lorite I J, Tornero S, et al. Regional calibration of Hargreaves equation for estimating reference ET in a semiarid environment. Agric Water Manage, 2006, 81: 257-281

29 Zhang Q, Wang S, Zeng J. On the non-rained land-surface water components and their relationship with soil moisture content in arid redion (in Chinese). Arid Zone Res, 2010, 27: 392-400 [张强, 王胜, 曾剑. 论干旱区非降水性陆面液态水分分量及其与土壤水分关 系. 干旱区研究, 2010, 27: 392-400]

30 Zhang Q, Hu X J, Wang S, et al. Some technological and scientific issues about the experimental study of land surface processes in Chinese Loess Plateau (LOPEX) (in Chinese). Adv Earth Sci, 2009, 24: 363-371 [张强, 胡向军, 王胜, 等. 黄土高原陆面过程试验研究 (LOPEX)有关科学问题. 地球科学进展, 2009, 24: 363-371]

31 Ke X X, Lin R N, Xu G C, et al. Manufacture of the large weighing lysimeter (in Chinese). Quart J Appl Meterol, 1994, 5: 344-353 [柯晓 新, 林日暖, 徐国昌, 等. 大型称重式蒸渗计的研制. 应用气象学报, 1994, 5: 344-353]

32 Zhang Q, Zhang Z X, Wen X M, et al. Comparisons of observational methods of land surface evapotranspiration and their influence factors (in Chinese). Adv Earth Sci, 2011, 26: 538-547 [张强, 张之贤, 问晓梅, 等. 陆面蒸散量观测方法比较分析及其影响因素研究. 地球科学进展, 2011, 26: 538-547]

33 Yang F, Zhang Q, Wang R, et al. Evapotranspiration measurement and crop coefficient estimation over a spring wheat farmland ecosystem in the Loess Plateau. PLoS One, 2014, 9: e100031

34 Yang X G, Zhang Q, Wang R Y, et al. Experimental study on surface energy balance over Loess Plateau of Middle Part Gansu in summer (in Chinese). Plateau Meteor, 2004, 23: 828-834 [杨兴国, 张强, 王润元, 等. 陇中黄土高原夏季地表能量平衡观测研究. 高原气象, 2004, 23: 828-834]

35 Zhang X D, Yang X G, Yang Q G. Study on laws of evapotranspiration of spring wheat in semi-arid region (in Chinese). Agric Res Arid Areas, 2004, 22: 63-66 [张旭东, 杨兴国, 杨启国. 半干旱区旱作春小麦耗水规律研究. 干旱地区农业研究, 2004, 22: 63-66]

36 Chen Z G, Bian L G, Lu L H. The comparison and application of the method to calculate atmospheric turbulence parameters near surface. Beijing: Chinese Academy of Meteorological Sciences. 2004 [谌志刚, 市林根, 陆龙弊. 近地层大气湍流参数求解方法的比较及其应 用. 硕士学位论文. 北京: 中国气象局气象科学研究院, 2004]

37 Xu Z W, Liu S M, Xu T R, et al. Comparison of the Gap Filling Methods of Evapotranspiration Measured by Eddy Covariance System (in Chinese). Adv Earth Sci, 2009, 29: 372-382 [徐自为, 刘绍民, 徐同仁, 等. 浴动相关仪观测蒸散量的插补方法比较. 地球科学进展, 2009, 29: 372-382]

38 Beven K. Sensitivity analysis of the Penman-Monteith actual evapotranspiration estimates. J Hydrol, 1979, 44: 169-190

39 Li Y L, Cui J H, Zhang T H. Comparative study on calculation methods of reference evapotranspiration (in Chinese). J Desert Res, 2002, 22: 372-376 [李玉霖, 崔建垣, 张铜会. 参考作物蒸散量计算方法的比较研究. 中国沙漠, 2002, 22: 372-376]

40 Wang J, Cai H J, Liu H Y. The estimation of evaportranspiration with Penman-Monteith and evaporator methods (in Chinese). Agric Res Arid Areas, 2002, 20: 67-71 [王健, 蔡焕杰, 刘红英. 利用 Penman-Monteith 法和蒸发皿法计算农田蒸散量的研究. 干旱地区农业研 究, 2002, 20: 67-71]

41 Juan C H, Shih S F. A lysimeter system for evapotranspiration estimation for wetland vegetation. Proc Soil Crop Sci Soc Fla, 1997, 56: 125-130 
42 Wright J L. New evapotranspiration crop coefficients. J Irrig Drain Division, 1982, 108: 57-74

43 Zhang Q, Wang S, Yang F L. The characteristics of dew formation/distribution and its contribution to the surface water budget over a semi-arid region in China. Bound-Layer Meteor, 2014, doi: 10.1007/s105476-014-9971-x

44 Li J, Liu Y F, Yang X G, et al. Studies on water vapor flux characteristic and the relationship with environment factors over a planted coniferous forest in Qianyanzhou station (in Chinese). Acta Ecol Sin, 2006, 26: 2456-2449 [李菊, 刘允芬，杨晓光，等. 千烟洲人工林水 汽通量特征及其与环境因子的关系. 生态学报, 2006, 26: 2456-2449]

45 Penman H L. Vegetation and Hydrogeology. Technical Comment No 53, Commonwealth Bureau of Soils, 1963, Harpenden, England

\title{
The influence of drought stress on spring wheat evapotranspiration and crop coefficients in semi-arid areas
}

\author{
ZHANG Qiang ${ }^{1,2,3}$, WANG WenYu ${ }^{4}$, YANG FuLin ${ }^{1,3}$, WANG XiaoPing ${ }^{1,3}$ \& ZHANG Liang ${ }^{1,3}$ \\ ${ }^{1}$ Institute of Arid Meteorology, China Meteorological Administration (CMA); Key Laboratory of Arid Climatic Change and Reducing Disaster of \\ Gansu Province; Key Open Laboratory of Arid Climatic Change and Disaster Reduction of CMA, Lanzhou 730020, China; \\ ${ }^{2}$ Gansu Provincial Meteorological Bureau, Lanzhou 730020, China; \\ ${ }^{3}$ College of Atmospheric Sciences, Lanzhou University, Lanzhou 730000, China; \\ ${ }^{4}$ Hubei Branch of CMA Training Centre, CMA, Wuhan 430074, China
}

An understanding of evapotranspiration, which is an important component of the surface water cycle, is critical in water resource management and planning, and in meeting the water requirements of agriculture and ecological systems. Although the methods for calculating crop evapotranspiration are advanced in humid areas, the understanding of evapotranspiration in semi-arid areas is developing because evapotranspiration is significantly affected by drought. The classic crop evapotranspiration model and crop coefficients cannot be applied to estimations of actual evapotranspiration, which is rarely studied now. In this study, we analyzed differences between lysimeter evapotranspiration and evapotranspiration that was estimated with Food and Agriculture Organization (FAO)-recommended crop coefficients. In addition, changes in evapotranspiration according to the degree of drought stress were analyzed with lysimeters, evaporating dishes, ultrasonic and eddy instruments, and conventional meteorological data in spring wheat fields in typical semi-arid zones of the Dingxi arid ecological environment comprehensive scientific experiment station, which is located in the Loess Plateau. The variations in spring wheat reference evapotranspiration/pan evaporation and actual evapotranspiration/pan evaporation according to the degree of drought stress and the effects of drought stress on the spring wheat crop coefficients in this area were studied. Because crop evapotranspiration is significantly affected by drought stress in semi-arid areas, the differences between lysimeter evapotranspiration and evaporation that was estimated with FAO-recommended crop coefficients were very significant. Crop coefficients were highly dependent on drought stress, and this dependence increased according to the reduction in the degree of drought stress. In addition, its sensitivity was significantly lower after the drought stress reached about 0.7 . In this study, crop coefficients that were modified during the crop development and exuberant period were far below the FAO-recommended values and the Kumar correction values. The modified crop coefficients were obviously closer to the actual observed values than the spring wheat evapotranspiration values that were estimated using other crop coefficient. The coefficient of linear fitting of the modified crop coefficient and the observations was 0.98 . The coefficient of determination was 0.45 , and the standard error was 0.85 . Therefore, the modified crop coefficient was effective for estimating crop evapotranspiration in semi-arid areas.

semi-arid areas, drought stress, crop evapotranspiration, crop coefficient, model improvement

doi: 10.1360/N972014-01116

\section{补充材料}

图 S1 观测站的地貌背景(a)和观测场环境(b)

图 S2 蒸渗仪观测的蒸散量与浴动相关法计算的蒸散量的比较

本文以上补充材料见网络版 csb.scichina.com. 补充材料为作者提供的原始数据，作者对其学术质量和内容负责. 